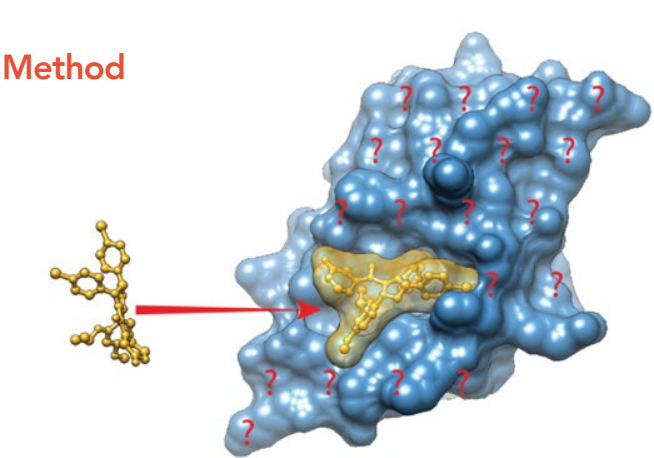

The challenge is to derive,
ligand complex structures

Without any protein assignment

: Without knowing the location of the binding site

With receptor (side chains \& backbone) and ligand flexibility wall growth and eventually leads to its degradation and to bacterial death. Some blocking drugs act in a similar way, by blocking the proteins responsible for DNA synthesis and hindering the growth of new cancerous tissue. Understanding how proteins involved in bacterial infections drugs, and how we can optimis these drugs to maximise their effects is currenty one of the crucial and most far-reaching issues in pharmacological research

and drug discovery.

\section{PROTEIN STRUCTURE}

DETERMINATION

macromolecules, composed of thousands of atoms, which are arranged in chains
of subuits, called amino acids. Proteins between the ligand and unknown methyl groups of $H D M X$. B Bottom: Ligand ' II mag,
build-up curves (right) versus the mixing time of the filtered NOESY experiments. run into problems with specific classes

increasing the rate of protein prodth

Understanding the nature of the interactions between proteins and ligands is a crucial issue in drug discovery.

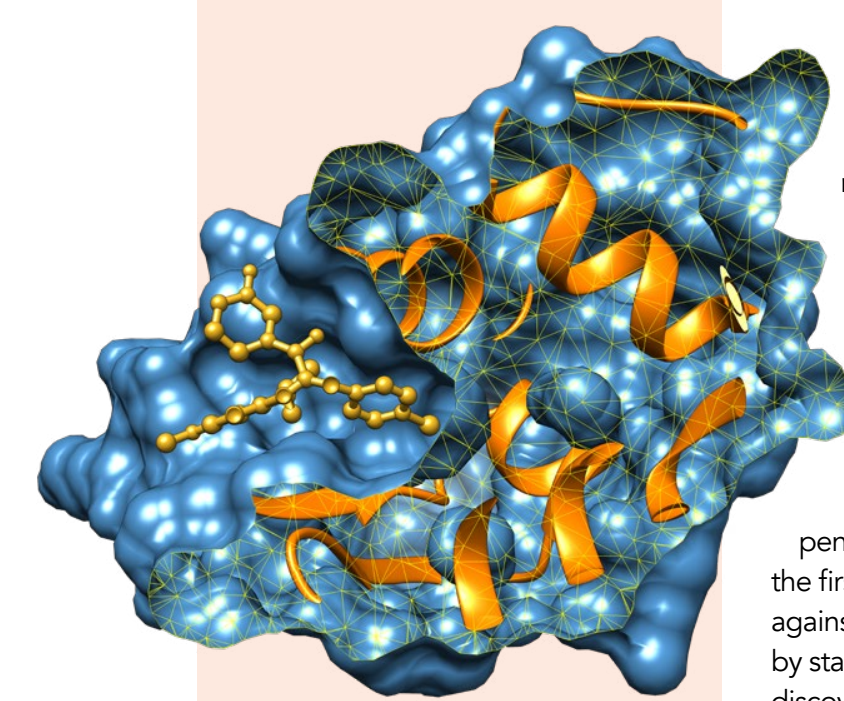

tissue. Similarly, cofactors are in a number of complex chemical reactions involved in the metabolic processes that keep an organism alive. Most drugs can themselves be classified as artificial ligands. They work in the same way as natural ligands, by binding to
specific sites in proteins and modifying the protein's function. A famous example of this is penicillin (an antibiotic, and one of against many bacterial infections caused by staphylococci and streptococci), discovered by Alexander Fleming in 1928 . senicilin acts by binding to receptor

fold into 3D structures, which are haracteristic of each protein and are connected at a fundamental level to the protein's function. Accurate knowledge of a protein's structure is the first step toward understanding how the protein works and how the chemico-physical processes that it carries out can be drug discovery typically starts by screening large databases of molecules or molecular fragments as potential ligands for a selected drug target. This approach is very error-prone and requires validation, e.g. by comparison to refined $3 \mathrm{D}$ complex structures. The gold standard in 3 D proten structure by X-ray crystallography, a powerfu

Experimental inter-molecular NOEs measured on a high field spectrometer: Top: ${ }^{3 C}$, , 1 N-filtered 2D-[H,'H-NOESY spectrum showing inter-molecular NOEs

approach that is frequently used ligand complexes, essentially at an atomic level. This technique requires crystalline samples, which can be expensive and time-consuming to obtain. X-ray crystallography can also of proteins, including membrane proteins and flexible receptors. The efficiency of X-ray crystallography can be improved substantially using the Molecular Replacement (MR) method, which relies on the existence of a previously resolved structure that is similar to the protein-complex under study. However, some classes of proteins (like membrane proteins and flexible receptors) remain beyond the capabilities of $X$-rays, and magnetic resonance (MMR), has been proposed as a potential alternative for these cases. NMR uses magnetic fields to gain information about the environment of each individual atom in a protein, for example, its chemical shift and dipoledipole interaction. The main limitation of NMR is that this technique requires long measurements and extensive data analysis. A recently proposed and fastdeveloping technique, cryo-electron microscopic, has also been shown to have great potential in drug discovery for large systems. Currently, however, X-ray crystallography and, to a lesser extent, MVR are by far the most widely used approaches to structural determination, structures have been resolved to date using these two complementary Dines to bridge the gap between $X$-rays and $N M R$, in order to expand the power and applicability of NMR and to make it a robust tool for drug discovery.

\section{NMR': A ZOOM}

\section{ON THE BINDING SITES}

The protein-ligand binding arises from very local interactions: ligands only bind to very specific sites in a protein, which typically involve only a relatively smal whe ratoms compared to the whole protein. Binding sites are just like enter and in which it can be stabilised by steric (i.e. depending on the shape

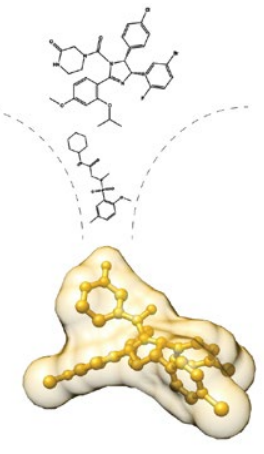

BINDIN

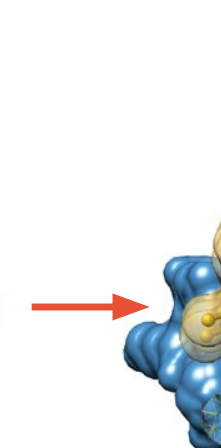

INTERMOLECULAR
COMMUNICATION

and size of the pocket), electrostatic chemical interactions. The global unaffected by the ligand binding Based on this obsenvation, Dr Orts and his collaborators have developed a powerful protocol to work out in great detail the structure of the protein-ligand binding site, using existing information about the global structure of the protein obtained in separate X-ray diffraction or liquid NMR measurements. This method, the Nuclear Magnetic Resonance Molecular replacement (NMRR), is based on solutionstate NMR structure determination, but it focusses on the structure of the bindingsite alone, rather han on the full protein and tedious alysis required to resolv 
the whole protein structure from NMR and effort required to obtain a tomically resolved structure of a binding pockt by using previously determined protein structures, from a couple of months to a couple of days. It can also be partially automated, and therefore it provides a natural tool for high-throughput workflows, which can be used to screen thousands of potential new drugs in sequence and to analyse the nature of their interaction with a target protein.

A typical NMR2 protein-ligand structure determination requires a preliminary preparation of the sample, in which either the protein or the ligand are isotopically substituted ( $C$, WN or selectively labelled (e.g. isoleucine, leucine and are then used to measure intra mo (ligand) and inter-molecular (ligand protein) atomic distances, which in tur provide a model of the ligand structure in the binding pocket To understand the exact nature of the ligand-protein interaction, protein structures from existing databases (obtained from X-ray
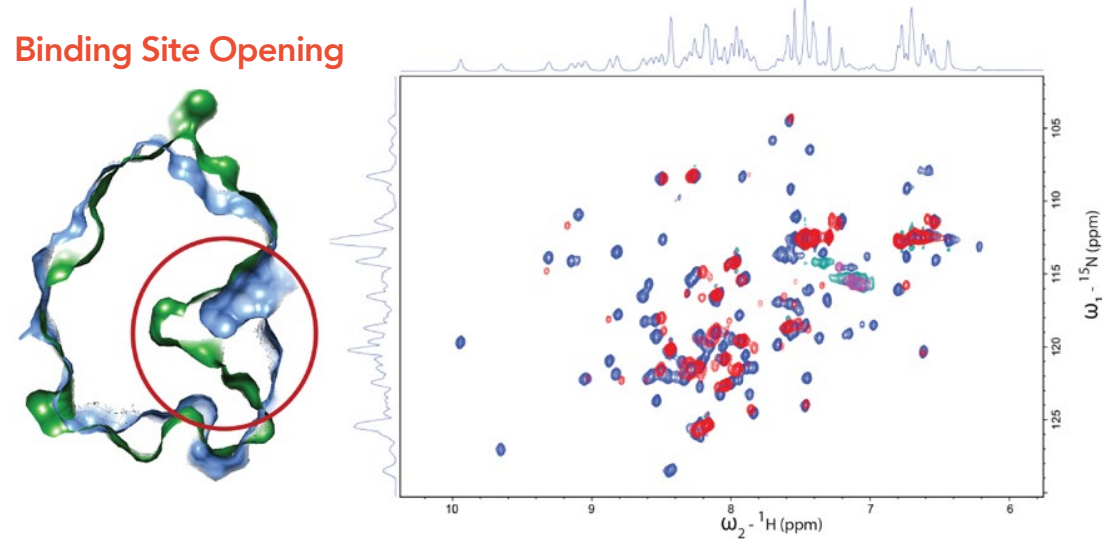

HDMX binds to 553 and inhibits the transactivation of $\mathrm{p5} 3$ target genes. $\mathrm{HDMX}$ binds to ligands via a
mechanism where disordered regions become structured. Left: Protein binding pocket (circled in red) opening upon 553 -peptidomimetic binding. Right: Effect on the protein NMR spectrum upon ligand

inputinformation The structures input information. The structures selected can be either that of the protein in the (homolog) proteins. The MMR $^{2}$ prograr then screens all possible assignment groups in the protein and calculates the protein-ligand complex structure for all options. At this stage, it is essential to reduce as much as possible the number of configurations to screen. This can be achieved by initially restricting the assignment groups in the protein to only 3 or 4 relevant ones. False assignments

The NMR ${ }^{2}$ methods reduce the time required to determine protein-ligand structures from months to a few days.

RMSD 0.9

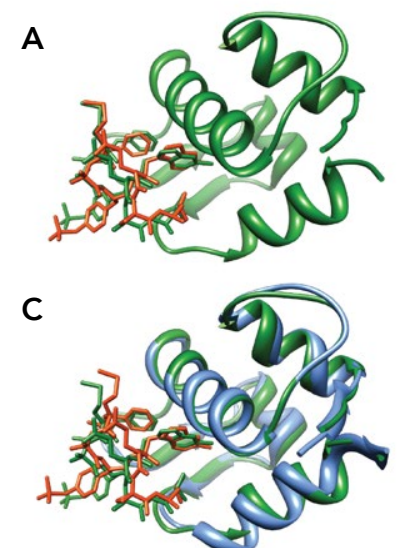

RMSD 1.1
RMSD $0.9 \AA$

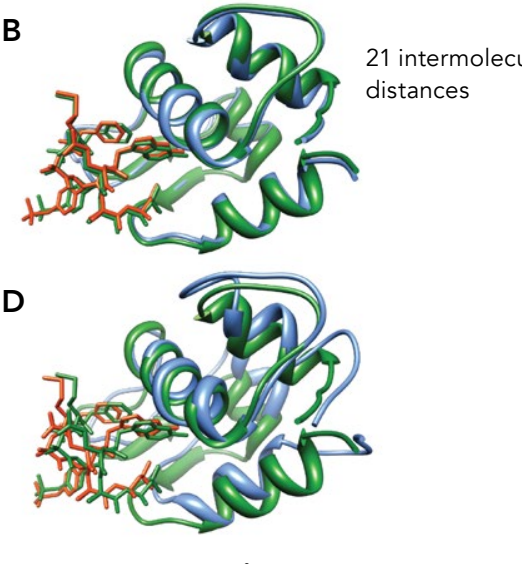

RMSD $1.8 \AA$

NMR2
structures of HDMX in complex with cmpd2 under different starting scenarios using either a)
the $3 D$ structure of the native holo-protein (3feal, b) the $X$-ray crystallography structure of HDMX in complex with a different ligand (3fel), c) a homology model of an $X$-ray crystallography structure of
HDM2 bound to a different ligand (2axi), ord) a homology model derived from the NMR structure

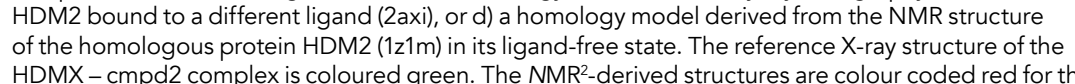
ligand and blue for the protein with the exception of the protein structure in a) because the structure
lidentical to the reference structure

can be ruled out using geometric considerations, based on the knowledge

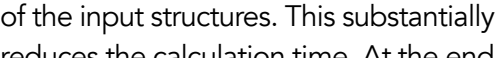
of this procedure, the resulting complex structures have to be analysed corefully coct potential errors aring from the unconstrained relaxation of the protein backbone during the refinement procedure. It is important that a sufficien number of inter-molecular distances are taken into account, typically at least 12 or 15. A high signal-to-noise in the NMR spectra and a good signal resolution

\section{A NEW TOOL FOR}

DRUG DISCOVERY

The NMR² method has been applied successfully to the resolution of various classes of protein-ligand complexes. binders or sull ligands hing strong determined with an accuracy of 0.9 1.5 angstroms relative to the reference structure. The applicability of $\mathrm{NMR}^{2}$ to complexes with ligands in fast exchange or weak affinity binding has also been demonstrated. In the case of the weak affinity binding complex HDM2-\#845, a new complex has been characterised, never observed before. The efficiency $N M R^{2}$ is well represented by the complex structure of SJ212-MDMX, which could be resolved at 1.35-angstrom accuracy within a day using a desktop computer. These are a few initial examples of the great potential of $\mathrm{NMR}^{2}$ in the study of protein-ligand interactions and protein function and they pave the way for its protocol for drug discovery.

\section{Behind the Research}

\section{Dr Julien Orts}

E: julien.orts@phys.chem.ethz.ch T: $+41(0) 446322864$ @JulienOrts

Research Objectives

Dr Orts and his collaborators have developed multidisciplinary approaches to study protein-small olecules complexes using NMR spectroscopy, X-ray crystallography and computational methods.

\section{Detail}

Dr Julien Orts

of Physical Chemistry

Swiss Federal Institute of Technology

HCI F217, Vladimir-Prelog-Weg 2

8093 Zürich

Switzerland

Bio from the Max Planck Institute and the European Molecular Biology Laboratory. He is currently a junior group leader at the ETH Zurich. He develops methods for structurebased drug design and demonstrated for the first time tha 3D structure determination of a proten ligand complex can be achieved from solution NMR data fully automatical

\section{Collaborators}

- May Marsh

- Roland Riek

- Dean Strotz

- Felix Torres

- Marielle Aulikki Wälti

\section{EIH}

Eidgenössische Technische Hochschule Zürich Swiss Federal Institute of Technology Zurich

\section{References}

Orts, I (2018). Research profile. [online]. Available at: https:// twitter.com/JulienOrts Interests.html [Accessed 28 Jan 2019.

Orts, J; Wälti MA; Marsh, M; Vera, L; Gossert, AD; Güntert, P; Riek, R. (2016). 'NMR-Based Determination of the 3D Structure of the Ligand-Proten' Interaction Ste without (3393.400.

Wälti MA; Riek, R.; Orts, J. (2017). 'Fast NMR-Based Determination of the $3 \mathrm{D}$ Structure of the Binding Site of Protein-Ligand Complexes with Weak Affinity Binders'. Angew. Chem. Int. Ed. 56, 5208-5211.

Wälti MA; Orts, J. (2018). 'The NMR² Method to Determine Rapidly the Structure of the Binding Pocket of a Protein4,12

\section{Personal Response}

The accuracy and efficiency of the NMR ${ }^{2}$ methods complexes. What are the remaining challenges of $N^{2}{ }^{2}$ that need to be addressed in order to make you method a robust and easy to use routine tool for high-

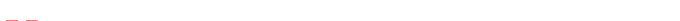

II Fragment-based drug discovery is becoming a major approach in both pharmaceutical companies and academic

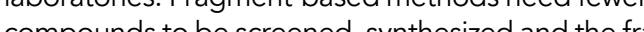
hits show usually high ligand efficiencies (potency per atom) Currently, no fast and robust NMR method can handle small fragments due to a lack of protons "probes". We need to develop the current NMR" method to simultaneously determine multiple struticully and protein complexes.

Having access to the structure of the binding site for each binder allows investigating chemical scaffolds that would thowlede as well as he dro ability of he heceptors. 